Problemfrakturen

\title{
Wieland Otto
}

Universitätsklinik für Unfall- und Wiederherstellungschirurgie, BG-Kliniken Bergmannstrost, Halle/Saale

\section{Behandlungsstrategie bei distalen Humerusfrakturen}

istale Humerusfrakturen sind Folge von verschiedenartigsten Verletzungsmechanismen und indirekter oder direkter Gewalteinwirkung. In Abhängigkeit davon entstehen sehr unterschiedliche Verletzungsbilder sowohl am Knochen selbst als auch an den umgebenden Weichteilen.

\section{Diagnostik und Klassifikation} tracht, ansonsten ist die operative Therapie indiziert. Dazu werden Kirschner-Drähte, Schrauben, Platten und Materialkombinationen angewendet, selten temporär der Fixateur externe. Nach Rekonstruktion der Gelenkrolle muss diese evtl.über zwischengeschaltete metaphysäre Fragmente mit dem Schaftfragment stabil verbunden werden. Als Zugänge dienen ein- oder beidseitige Schnittführungen oder, am häufigsten, die dorsale Schnittführung mit Osteotomie des Olekranons. Postoperativ ist eine frühzeitige Physiotherapie zwingend notwendig, um Einsteifungen zu vermeiden.Zur Prophylaxe drohender ektoper Ossifikationen sind in aller Regel Gaben von nichtsteroidalen Antirheumatika und/oder die postoperative Röntgenbestrahlung indiziert. Arthrolysen sind erst nach Ausreifung ektoper Ossifikationen und nach abgeschlossener Frakturheilung angezeigt, dann optional mit der abschließenden Metallentfernung kombiniert.

\section{Schlüsselwörter}

Verletzungsmechanismus · Frakturklassifikation · Behandlungsgrundsätze · Nachsorge · Korrektureingriffe

Die Diagnose einer distalen Humerusfraktur ergibt sich aus

- der Unfallanamnese,

- dem klinischen Befund mit evtl. Fehlstellung und gestörter Funktion sowie

- Schmerzen.

In Abhängigkeit vom Schweregrad der Verletzung kommen erkennbare Weichteilschäden hinzu.

Mit Hilfe der Röntgendarstellung in 2 Ebenen (beim Verdacht auf eine distale Humerusfraktur immer senkrecht zum Humerusschaft!) sind meist eine zweifelsfreie Identifizierung der Verletzung und eine Schweregradbeurteilung möglich. Nur selten sind zusätzliche Schrägaufnahmen erforderlich. Bei multifragmentären Frakturen, v. a. unter Einbeziehung der Trochlea humeri, können CT-Darstellungen weitere Informationen für die Therapieplanung geben. $\mathrm{Zu}$ achten ist insbesondere auf so genannte okkulte Frakturlinien oder „Fissuren“, bei deren Übersehen Probleme bei der Durchführung und
Die Schweregradbeurteilung der Fraktur erfolgt durch die AO-Klassifikation in

- extraartikuläre Frakturen (13-A),

- partiell intraartikuläre Frakturen (13-B) und

- komplett intraartikuläre Frakturen (13-C).

Die immer noch gebräuchliche „klinische" Einteilung in dem Erfolg der Therapie entstehen können.
- suprakondyläre Frakturen,

- mono- oder transkondyläre Frakturen und schließlich

- trans- und suprakondyläre Frakturen

folgt diesen Einteilungsprinzipien in weitgehender Annäherung.

Nur selten und eher bei direkter Gewalteinwirkung treten höhergradige Weichteilschäden, große Wunden oder ausgedehnte Gewebeverluste auf. Derartige Weichteilschäden sind für das Behandlungsergebnis und die wiederzuerlangende Ellbogengelenkfunktion immer von größter Bedeutung. Bei Weichteilverlusten ist wegen der Frage des freien Lappentransfers eine frühe Einschaltung plastischer Chirurgen notwendig. An Verletzungen der Nn. ulna-

\footnotetext{
Prof. Dr. Wieland Otto

Universitätsklinik für Unfall-

und Wiederherstellungschirurgie,

BG-Kliniken Bergmannstrost,

Merseburger Straße 165, 06112 Halle/Saale,

E-Mail:wieland.otto@bergmannstrost.de,

Phone:0345-1326325, Fax:0345-1326326
} 
Trauma Berufskrankh

$2003 \cdot 5$ [Suppl 1]: S17-S21 @ Springer-Verlag 2003

\section{W. Otto}

\section{Treatment strategy of distal humerus fractures}

\section{Abstract}

Fractures of the distal humerus are caused through direct or indirect injury mechanisms. The types of fractures and soft tissue lesions vary widely, depending on the mechanism of injury. The aims of therapy are painless joint function and stability. Conservative treatment is only indicated in stable fracture types in childhood. In all other cases operative therapy is mandatory. Kirschner wires, screws, plates and different combinations are implanted, and occasionally an external fixator is used temporarily. After reconstruction the trochlea has to be securely fixed to the shaft fragment, if necessary by means of interposed metaphyseal fragments. Operative approaches include incisions made from one or both sides, but in most cases a dorsal incision is used with osteotomy of the olecranon. Early postoperative physiotherapy is mandatory to avoid contraction of the elbow joint. Nonsteroidal anti-inflammatory medications and/or postoperative X-ray irradiation are normally indicated to prevent ectopic ossifications. Arthrolysis should be carried out only when such heterotopic ossifications are fully mature and can be combined with removal of the osteosynthesis material if fracture healing is complete.

\section{Keywords}

Trauma mechanism - Fracture classification . Treatment principles $\cdot$ Aftercare $\cdot$ Corrective operations

\section{Problemfrakturen}

ris, medianus und radialis sowie der A. brachialis bzw. cubitalis und ihrer beiden Abgänge, den Aa. radialis und ulnaris, ist grundsätzlich zu denken. Überprüfung der Sensibilität und der motorischen Handfunktionen sowie des Pulsstatus gehören daher zur klinischen Erstuntersuchung und bedürfen ggf. der kurzfristigen Wiederholung!

\section{Behandlungsgrundsätze}

Behandlungsziel bei distalen Humerusfrakturen, v. a. mit Gelenkbeteiligung, muss sein:

- anatomische Rekonstruktion unter besonderer Beachtung von Trochlea, Capitulum, Fossae coronoidea und olecrani und der physiologischen Anteflexion von $15^{\circ}$

- schmerzfreie Funktion, bezogen auf Beweglichkeit und Stabilität,

- Wiederherstellung einer vollen Belastbarkeit im Sinn der Beanspruchbarkeit im täglichen Leben und im Beruf

Nicht dislozierte und stabile Frakturen können grundsätzlich konservativ behandelt werden. Davon wird v. a.im Kindesalter bei den reinen suprakondylären Hyperextensionsfrakturen mit erhaltenem dorsalem Periost gern Gebrauch gemacht.

Im Erwachsenenalter wird nur in Ausnahmefällen unter Beachtung vieler Begleitumstände konservativ therapiert. Hier ist ganz überwiegend die operative Versorgung mit dem Ziel einer übungsstabilen Osteosynthese und frühen funktionellen Behandlung indiziert. Als problematische Verletzungen hinsichtlich der Operationsindikation und -taktik sowie des richtigen Zeitpunkts sind zu nennen: die A.3-, B3- und alle C-Frakturen sowie Gelenkbrüche bei schwerer Osteoporose, begleitendem schwerem Schädel-Hirn-Trauma und die Verletzung als Teil eines Polytraumas.

Allgemeine Probleme, die bei der Therapieentscheidung zu berücksichtigen sind, ergeben sich bei Alkoholikern, dementen Patienten, sehr alten Menschen und eingeschränkter bzw. fehlender Compliance $[5,9]$. Hier muss u. U. eher an eine konservativ-immobilisierende Therapie gedacht werden.

\section{Operative Therapie}

Das Behandlungsergebnis bei distalen Oberarmfrakturen hängt sehr stark von verschiedenen Faktoren ab, die bei der strategischen und taktischen Planung operativer Behandlungsmaßnahmen deshalb immer zu berücksichtigen sind.

\section{Operationszeitpunkt}

Bei offenen Verletzungen ist immer die notfallmäßige Versorgung angezeigt, bei hohem Schweregrad ggf. geplant schrittweise zu komplettieren. Bei geschlossenen Verletzungen sollte die definitive Behandlung frühestmöglich erfolgen, da jede Form der vorläufigen Ruhigstellung oder Transfixation zu einer verstärkten Einsteifungstendenz im weiteren Verlauf führt. Des Weiteren ist bekannt, dass bei verzögerten Operationen eine erhöhte Rate verstärkt ausgeprägter ektoper Ossifikationen zu erwarten ist.

\section{Klassifikation, Lagerung und operative Zugänge}

Einfache Frakturtypen können durch uni- oder bilaterale seitliche Zugänge gut therapiert werden. Dann ist in der Regel die Lagerung des Patienten auf dem Rücken mit Auslagerung des Arms auf dem Armtisch möglich. Hautschnitt und Zugang werden direkt über dem verletzten Epikondylus oder Condylus humeri gelegt. Bei beidseitigem Zugang

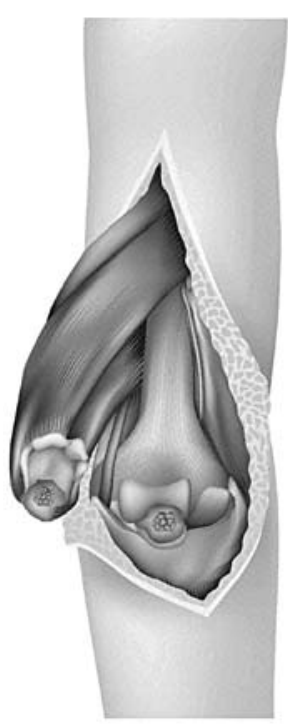

Abb. $1 \Delta$ Dorsaler Zugang zum distalen Humerus mit Olekranonosteotomie [3] 

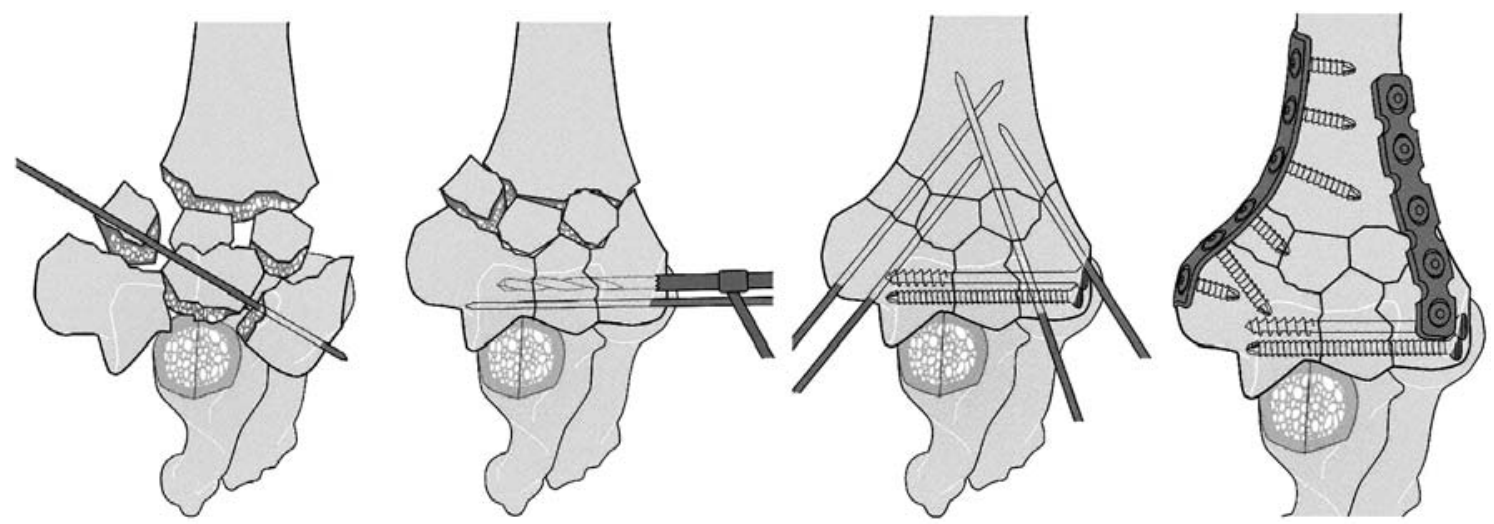

Abb. $2 \Delta$ Rekonstruktionsschritte bei mehrfragmentärer trans- und suprakondylärer distaler Humerusfraktur: Rekonstruktion von Trochlea und Capitulum, Verschraubung des Gelenkblocks, Reposition und temporäre Fixation zum Schaftfragment, Doppelplattenosteosynthese [3]

ist auch ein dorsaler Hautschnitt möglich mit in der Tiefe dann beidseitiger Umgehung der Trizepssehne und bilateralem Zugang zum Knochen.

Für alle komplexeren Frakturen, bei denen der dorsale Zugang mit Olekranonosteotomie (Abb. 1) erforderlich wird, ist die Lagerung des Patienten auf dem Bauch oder auf der Seite vorzunehmen. Der verletzte Arm wird ellbogengelenknah am Humerus durch eine Rolle unterstützt, sodass der Unterarm frei pendelnd abwärts hängt und das Ellbogengelenk nach Möglichkeit bis zu $120^{\circ}$ gebeugt werden kann. Damit ist intraoperativ eine ausreichende Übersicht über das gesamte Gelenk gewährleistet.

Zusammenfassend lässt sich sagen:

- A.1- und in der Regel alle B-Frakturen sind durch einseitige seitliche Zugänge gut zu reponieren und zu fixieren.

- Für A.2-, A.3- und C.1-Frakturen können bilaterale seitliche Zugänge notwendig und ausreichend sein.

- C.2- und insbesondere C.3-Verletzungen bedürfen in aller Regel des ausgedehnten dorsalen Zugangs mit Olekranonosteotomie.

\section{Repositionsmanöver}

Bei den komplexeren Frakturen empfiehlt es sich, zunächst die Fragmente der Gelenkrolle und des Capitulum humeri wieder exakt zusammenzufügen. Dabei sind möglichst alle Fragmente wieder einzubauen. Bei eventuellem Fragmentverlust sind Defekte durch Spongiosa aufzufüllen. Dabei soll unmittelbare Nähe zum Gelenkspalt mög- lichst vermieden werden. Die Trochlea wird dann in sich mittels KirschnerDrähten und Kompressions- oder Stellschrauben stabilisiert. Im 2. Schritt werden allfällige metaphysäre Zwischenfragmente zum Schaftfragment hin reponiert und temporär fixiert. Schließlich wird der Gelenkblock an den proximalen Fragmentverbund angepasst und die definitive Stabilisierung in aller Regel mit Platten und Schrauben, seltener mit Schrauben allein, durchgeführt. Beim Repositionsmanöver kann die Verwendung von spitzen Repositionszangen und temporär eingebrachten KirschnerDrähten hilfreich sein $[2,3,8]$. Eher selten wird ein Distraktor vom Humerusschaft auf die proximale Ulna benötigt, um für die ausreichende Länge und Ruhe im Frakturgebiet zu sorgen. Auch ein temporär angelegter Fixateur externe kommt ggf. dafür in Frage.

Sorgfältig zu vermeiden ist jede zusätzliche Devastierung der Fragmente. Größte Aufmerksamkeit ist der Vermeidung von Schäden an den Nn. ulnaris, radialis und ggf. medianus zu schenken. An den beugeseitig sehr knochennahen Verlauf der A. brachialis/cubitalis ist immer zu denken!

\section{Osteosynthesetechnik}

Wie bereits erwähnt, sind zur definitiven Stabilisierung v. a. Schrauben und Platten (LCDCP und Rekonstruktionsplatten) bzw. Kombinationen davon in Anwendung (Abb. 2).

Nicht selten werden zusätzlich Kirschner-Drähte als Rotationsstabilisatoren oder zur Adaptation kleiner, nicht stabilitätsvermittelnder Fragmente eingesetzt. Die extreme Ausnahme bildet eine vorübergehende oder länger dauernde zusätzliche Stabilisierung mittels gelenküberbrückendem Fixateur externe bei hochgradiger Zertrümmerung und evtl.begleitendem ausgeprägtem Weichteilschaden, der eine frühfunktionelle Therapie ohnehin nicht möglich werden lässt [7] (Abb. 3).

Zusammengefasst kann von Folgendem ausgegangen werden:

- Bei A.1- und den meisten B-Frakturen ist eine Schraubenosteosynthese, evtl. mit einem Antirotations-KirschnerDraht kombiniert, ausreichend und erfolgreich.

- A.2- und A.3-Frakturen werden mit einoder beidseitiger Plattenosteosynthese sinnvoll therapiert.

- Bei den C.1-Verletzungen können alleinige Schraubenosteosynthesen bei guter Knochenqualität zum Erfolg führen.

- Bei den C.2- und C.3-Frakturen kommen ein- oder beidseitige Plattenstabilisierungen in Kombination mit Schrauben und evtl. Kirschner-Drähten zum Einsatz. Insbesondere bei den C.3-Verletzungen ist bei der Doppelplattenosteosynthese darauf zu achten, dass die radialseitig angelegte Platte dorsal und weit nach distal reichend angebracht wird. Die ulnare Platte sollte dann in etwa $90^{\circ}$ zur radialen Platte ulnarseitig dem Humerus und dem Epicondylus ulnaris angelegt werden (Abb. 2,3). Auf diese Weise ist ein Höchstmaß an Biegebeanspruchbarkeit der Frakturzone zu erwarten [11]. Am Ende einer solchen Operation erfolgt die 0steosynthese 


\section{Problemfrakturen}
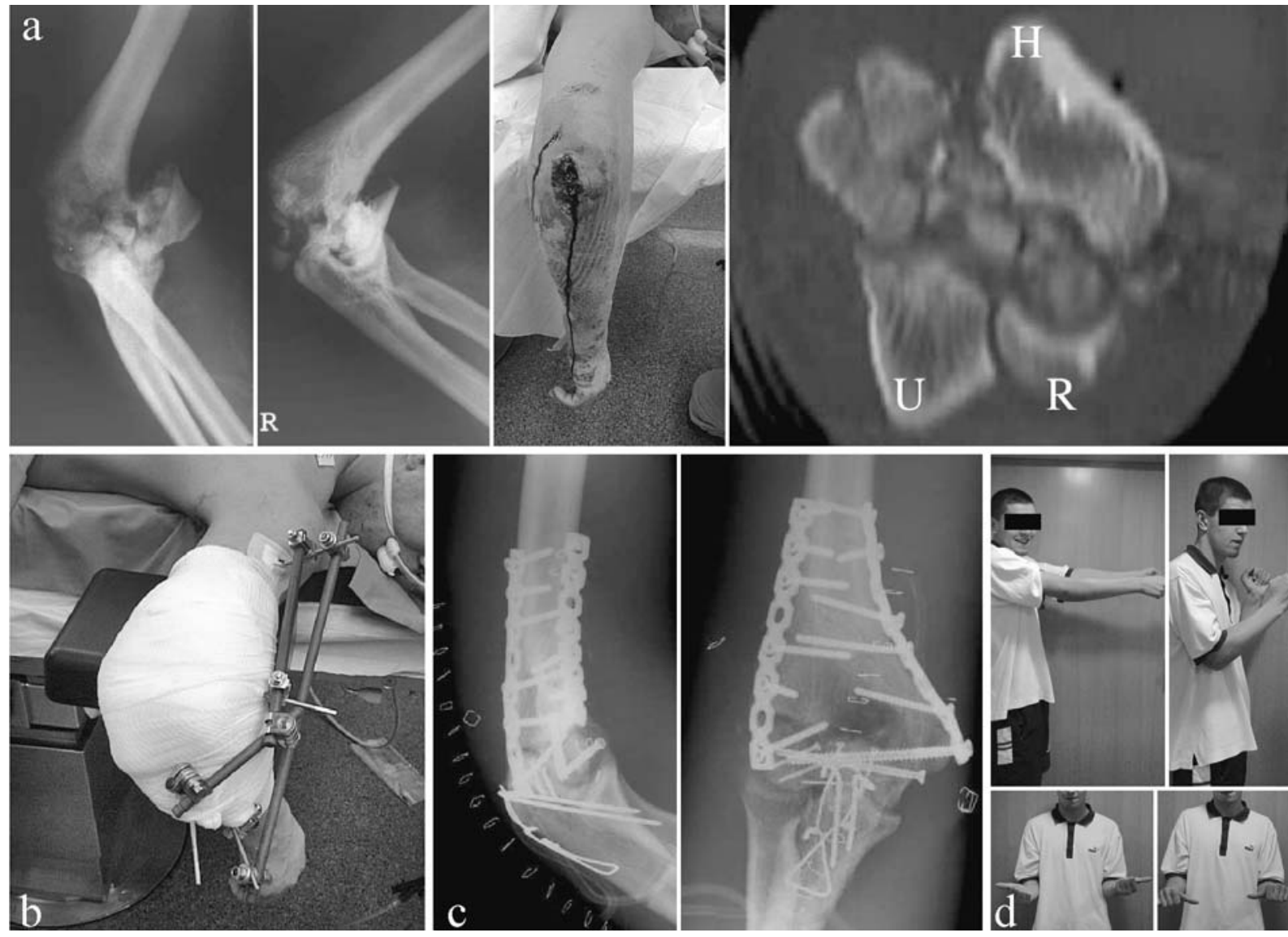

Abb. 3a-d $\Delta$ 19-Jähriger, als Pkw-Beifahrer eine zweitgradige offene C.3-Fraktur des distalen Humerus erlitten (a), primäre Weichteilsanierung und temporäre Stabilisierung mit einem gelenküberbrückenden Fixateur externe (b), frühsekundäre offene Reposition und Doppelplattenosteosynthese (c), sehr gute Beweglichkeit 3 Monate nach dem Unfall (d)

des osteotomierten Olekranons mit einer typischen Zuggurtung (Abb. 3).Der Wundverschluss darf nicht erzwungen werden. Insbesondere bei offenen Frakturen kann eine temporäre Deckung mit Kunsthaut oder durch Vakuumversiegelung erfolgen. Der Sekundärverschluss wird dann in aller Regel nach 48-72 $\mathrm{h}$ zu erreichen sein.

\section{Nachbehandlung}

Postoperativ erfolgen zur Sicherung der Wundheilung leichte Hochlagerung und Ruhigstellung mit einer Oberarmlonguette. Unter Abnahme der Longuette wird jedoch vom ersten postoperativen Tag an die isotonische Bewegungstherapie durchgeführt. Dabei ist der Einsatz von Motorschienen (CPM) aus unserer Sicht sehr zu empfehlen [12]. Sobald sich die Wundheilung als unproblematisch abzeichnet, kann die Longuette abgenommen und zu assistierten aktiven Be- wegungsübungen übergegangen werden. Diesen schließt sich eine abgestufte Physiotherapie unter Einbeziehung physikalischer Maßnahmen und der Ergotherapie an. Im späteren Rehabilitationsfortgang kommt Sporttherapie hinzu.

Zur unmittelbaren Weiterbehandlung nach der Operation gehört auch die Prophylaxe gegen ektope Ossifikationen. Diese wird mit nichtsteroidalen Antirheumatika (Diclophenac oder Indometacin 2-mal $50 \mathrm{mg}$ /Tag über $2-3$ Wochen) und nach Einzelfallentscheidung durch Röntgenbestrahlung unmittelbar bzw. frühzeitig postoperativ durchgeführt. In der Regel werden 2-mal 2,55 Gy am 1. und am 3. postoperativen Tag verabfolgt.

\section{Komplikationen und Fehlergebnisse}

Komplikationen können in Form eines oberflächlichen oder in die Tiefe gehen- den Infekts auftreten, v. a. bei Verletzungen mit ausgeprägtem Weichteilschaden, häufig dann von der Primärkontamination oder einer Wundrandnekrose bei erzwungenem Wundverschluss ausgehend. Intraoperative Läsionen oder Irritationen der Nn. ulnaris, radialis und medianus kommen vor, sind in der Regel jedoch rückbildungsfähig, sofern nicht echte strukturelle Beschädigungen erfolgt sind. Sekundäre Nervenirritationen können durch narbig-bindegewebige oder knöcherne Einhüllungen und Einengungen der Nerven entstehen. Hier sind operative Revisionen angezeigt.

Eher als Fehlergebnis oder Spätkomplikation sind die starke Einsteifung des Gelenks und das Auftreten ausgeprägter ektoper Ossifikationen mit Behinderung der Beweglichkeit zu bezeichnen. Ursache einer eingeschränkten Gelenkbeweglichkeit kann auch die Fehllage des eingebrachten Osteosyn- 
thesematerials sein, wie z. B. das Durchziehen der Fossa olecrani oder coronoidea durch eine fehlerhaft eingebrachte Schraube.

Pseudarthrosen entstehen aufgrund avitaler bzw. zu weitgehend devastierter Fragmente, verbliebener übermäßiger Fragmentdiastasen und fehlerhafter Osteosynthesetechnik. Das in den meisten Fällen mit der Ausbildung von Pseudarthrosen zu beobachtende Brechen von Implantaten ist daher nicht als „Implantatversagen “ $\mathrm{zu}$ bewerten, sondern eher Folge des Versagens der operativen Therapie insgesamt oder einer nicht korrekten Vorgehensweise während der Operation [6].

\section{Klinisches Ergebnis und Bewertung}

Bei den komplexeren Verletzungen am distalen Humerus verbleiben trotz bester operativer Therapie in aller Regel gewisse funktionelle Einschränkungen, insbesondere die Streckung und Beugung des Ellbogengelenks betreffend. Diese müssen jedoch im täglichen oder beruflichen Leben nicht zu Beeinträchtigungen führen. Deshalb bewertete $z$. B. Cassebaum [1] in seinem Score einen Bewegungsumfang von $\mathrm{o} / 15 / 125^{\circ}$ bei Streckung und Beugung als sehr gutes Resultat. Noch mit einer Einschränkung auf $0 / 40 / 120^{\circ}$ gilt das Ergebnis als gut. Ist die Beugefähigkeit auf $<110^{\circ}$ eingeschränkt, ist nach Cassebaum von einem mäßigen Resultat zu sprechen. In ähnlicher Weise wird die Minderung der Erwerbsfähigkeit im BG-lichen Begutachtungswesen von den Bewegungseinschränkungen abhängig gemacht. Der vollständige Verlust von Streck- und Beugefunktion bei $o^{\circ}$ wird rechts mit
50\%, links mit 40\% MdE bewertet, die Einsteifung bei $30^{\circ}$ mit 40 bzw. $30 \%$ und jene bei $120^{\circ}$ mit 30 bzw. $20 \%$ (Gebrauchshand bzw. Beihand) $[4,10]$.

Weniger häufig betroffen von verbleibenden Funktionsstörungen ist nach distalen Humerusfrakturen die Umwendebewegung von Unterarm und Hand. Der vollständige Verlust in Mittelposition wird rechts mit 30, links mit $20 \%$ bewertet. Die Einsteifung in submaximaler Pronation wird mit 20 bzw. $10^{\circ}$ und jene in submaximaler Supination mit 40 bzw. $30 \%$ eingestuft.

\section{Fazit für die Praxis}

Folgende Punkte sind zu beachten: Die Behandlung der distalen Humerusfrakturen ist mit Ausnahme bestimmter Frakturtypen im Kindesalter in aller Regel operativ durchzuführen.

Zum Einsatz kommen Schrauben, geeignete Platten und Kirschner-Drähte, häufig in Kombination miteinander.

Bei einfachen Frakturtypen genügen seitliche Zugänge. Komplexe Frakturen werden über dorsale Zugänge mit Olekranonosteotomie angegangen. Die operative Behandlung komplexer Frakturen kann sehr schwierig sein, viel Erfahrung erfordern und höchste Ansprüche an Mannschaft und Equipment stellen!

Bei höhergradigen Verletzungen (Fraktur und/oder Weichteilschaden) ist immer die frühzeitige (sofortige!) Verlegung in ein geeignetes Zentrum zu erwägen! Ein günstiger Therapieerfolg kann nur erwartet werden, wenn nach korrekter 0steosynthese mit funktionsstabilem Resultat eine sofortige funktionelle Nachbehandlung konsequent durchgeführt wird. Dazu gehört auch die Prophylaxe gegen ektope Ossifikationen medikamentös und/oder mittels Röntgenbestrahlung. Auch bei korrekt abgelaufener Therapie und radiologisch günstigem Resultat verbleiben in aller Regel gewisse funktionelle Einschränkungen, die gutachterlich angemessen zu berücksichtigen sind.

\section{Literatur}

1. Cassebaum WH (1969) Open reduction of T \& $Y$ fractures of the lower end of the humerus. J Trauma 9: 915-925

2. Heim U, Pfeiffer KM (Hrsg) (1988) Periphere Osteosynthesen.Springer, Berlin Heidelberg New York

3. Holdsworth BJ (2000) Humerus: distal. In: Rüedi TP, Murphy WM (eds) AO principles of fracture management. Thieme, Stuttgart New York

4. Izbicki W, Neumann N, Spohr H (Hrsg) (1992) Unfallbegutachtung. de Gruyter, Berlin New York

5. John H, Rosso R, Neff U, Bodoky A, Regazzoni $P$, Harder F (1993) Distale Humerusfrakturen bei Patienten über 75 Jahre. Langzeitergebnisse der Osteosynthese. Helv Chir Acta 60:219-224

6. Kundel K, Braun W, Wieberneit J, Rüter A (1996) Intraarticular distal humerus fractures. Factors affecting functional outcome. Clin Orthop 332: 200-208

7. Mostafavi HR, Tornetta P 3rd (1997) Open fractures of the humerus treated with external fixation.Clin Orthop 337: 187-197

8. Müller ME, Allgöwer M, Schneider R, Willenegger $\mathrm{H}$ (Hrsg) (1992) Manual der Osteosynthese. Springer, Berlin Heidelberg New York

9. Pereles TR, Koval KJ, Gallagher M, Rosen $\mathrm{H}$ (1997) Open reduction and internal fixation of the distal humerus: functional outcome in the elderly.J Trauma 43:578-584

10. Schönberger A, Mehrtens G, Valentin H (1998) Arbeitsunfall und Berufskrankheit. Schmidt, Berlin

11. Self J, Viegas SF, Buford WL Jr, Patterson RM (1995) A comparison of double-plate fixation methods for complex distal humerus fractures. J Shoulder Elbow Surg 4: 10-16

12. Soffer SR, Yahiro MA (1990) Continuous passive motion after internal fixation of distal humerus fractures. Orthop Rev 19:88-93 